\footnotetext{
${ }^{14}$ C. F. Coll, III, A. B. Harris, and A. J. Berlinsky, Phys. Rev. Lett. 25, 858 (1970).

${ }^{15} \mathrm{~J}$. C. Raich and R. D. Etters, Solid State Commun. 7, 1031 (1969).

${ }^{16}$ R. J. Lee, J. C. Raich, and R. D. Etters, Solid State Commun. 8, 1803 (1970).

${ }^{17}$ B. Strie $\bar{b}$, H. B. Callen, and G. Horwitz, Phys. Rev. 130,1798 (1963).

${ }^{18}$ T. Fujishiro, F. Takano, and T. Oguchi, J. Phys. Soc. Jap. 19, 1666 (1964).

${ }^{19}$ T. Morita and T. Tanaka, Phys. Rev. 145, 288 (1966).
}

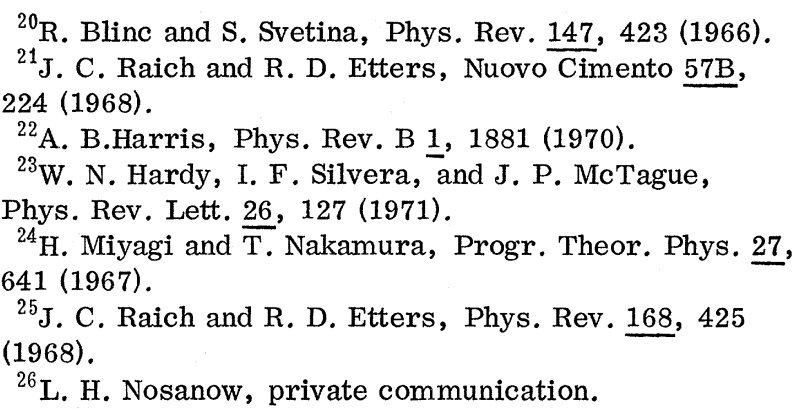

${ }^{20}$ R. Blinc and S. Svetina, Phys. Rev. 147, 423 (1966).

${ }^{21} \mathrm{~J}$. C. Raich and R. D. Etters, Nuovo Cimento 57B 24 (1968).

A. B.Harris, Phys. Rev. B 1 Phys. Rev. Lett. 26, 127 (1971).

${ }^{24}$ H. Miyagi and T. Nakamura, Progr. Theor. Phys. 27, (1968)

${ }^{26}$ L. H. Nosanow, private communication.

\title{
Noncollinear Spin Arrangement in Ultrafine Ferrimagnetic Crystallites
}

\author{
J. M. D. Coey* \\ Department of Physics, University of Manitoba, Winnipeg 19, Canada
}

(Received 30 August 1971)

\begin{abstract}
Mössbauer-effect measurements on extremely small ( $\sim 0 \AA \AA)$ crystallites of $\gamma-\mathrm{Fe}_{2} \mathrm{O}_{3}$ show that the spin configuration differs from the Neel type found in large crystallites. It is proposed that the ions in the surface layer are inclined at various angles to the direction of the net moment.
\end{abstract}

It is known that crystallites of $\gamma-\mathrm{Fe}_{2} \mathrm{O}_{3}$, small enough to be superparamagnetic, have a saturation magnetization which decreases abruptly with decreasing particle size. ${ }^{1}$ It has been variously proposed that low moments in $\gamma-\mathrm{Fe}_{2} \mathrm{O}_{3}$ can be explained by nonmagnetic grain boundaries ${ }^{1,2}$ or the presence of hydrogen in the lattice. ${ }^{3}$ We have established that neither explanation can account for the magnetization of a sample of ultrafine $\gamma-\mathrm{Fe}_{2}-$ $\mathrm{O}_{3}$, and that such small ferrimagnetic particles have a different spin structure from bulk material. Values of the saturation magnetization measured in bulk materials cannot, therefore, be expected to apply to very small particles.

The sample was prepared by precipitation from a solution of ferrous and ferric chloride, and subsequent oxidization in air. ${ }^{4}$ It was identified as $\gamma-\mathrm{Fe}_{2} \mathrm{O}_{3}$ from its Mössbauer spectra and its $\mathrm{x}$-ray powder pattern which showed eleven lowangle spinel-structure lines. Examination of the powder in an electron microscope showed that it consisted of agglomerates $10^{3}-10^{4} \AA$ across, which were composed of roughly spherical particles, 50-75 $\AA$ in diameter.

The material was superparamagnetic at room temperature in both magnetization and Mössbauer measurements, and the shape of the spectrum corresponded to a relaxation time of $2 \times 10^{-9} \mathrm{sec}$. An effective anisotropy constant $K=1.2 \times 10^{6} \mathrm{erg} /$ $\mathrm{cm}^{3}$ was deduced. ${ }^{4,5}$ The stable ferrimagnetic fraction was obtained from the ratio of the rema- nence to the saturation magnetization, as a function of temperature, and the particle-size distribution of Fig. 1 was obtained. $\int_{0}^{D} \varphi(D) d D$ is the fraction of the sample composed of particles with a diameter less than $D$. According to this distribution, the average particle size is $59 \AA$, and $40 \%$ of the iron ions lie in the surface layer.

$\gamma-\mathrm{Fe}_{2} \mathrm{O}_{3}$ is usually supposed to have the formula

$$
\left(\mathrm{Fe}^{3+}\right)\left[\mathrm{Fe}_{5 / 3}{ }^{3+} \square_{1 / 3}\right] \mathrm{O}_{4}^{2-} \text {, }
$$

where the different brackets denote the $A$ and $B$ sites of the spinel lattice. Iron ions on the two sites are aligned antiparallel, ${ }^{6}$ and the formula gives a spin-only moment of $87.4 \mathrm{emu} / \mathrm{g}$. The moment of our sample, obtained by extrapolation

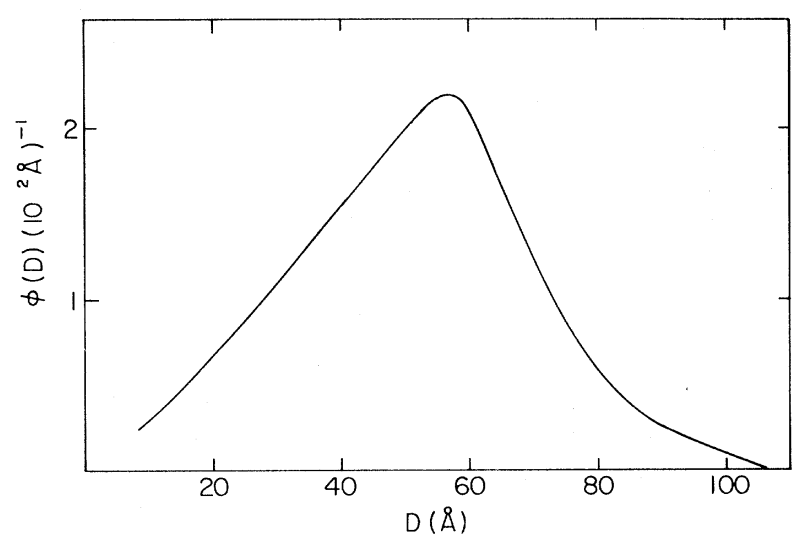

FIG. 1. Particle-size distribution of ultrafine $\gamma-\mathrm{Fe}_{2} \mathrm{O}_{3}$ crystallites. 


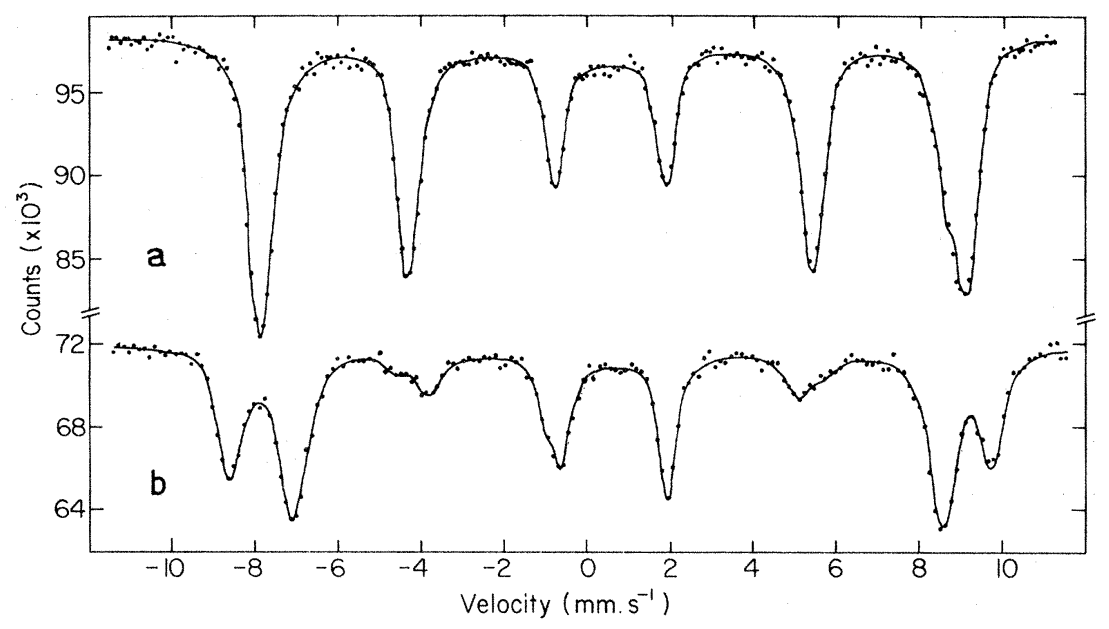

FIG. 2. Mössbauer spectra of ultrafine $\gamma-\mathrm{Fe}_{2} \mathrm{O}_{3}$ crystallites at $5^{\circ} \mathrm{K}$ : (a) without and (b) with an applied field of 50 kOe. Least-squares fits of the data by twelve Lorentzians are shown.

to $1 / H=0$ at $4.2^{\circ} \mathrm{K}$, was $59 \mathrm{emu} / \mathrm{g}$.

Mössbauer spectra taken at $5^{\circ} \mathrm{K}$ in the absence and presence of an applied field of $50 \mathrm{kOe}$ are shown in Fig. 2. The spectrum of Fig. 2(a) differs from that of bulk $\gamma-\mathrm{Fe}_{2} \mathrm{O}_{3}$ only in that the linewidths are $0.2 \mathrm{~mm} / \mathrm{sec}$ greater. There is no indication of any "nonmagnetic" iron, be it paramagnetic or antiferromagnetic, nor is there any significant amount of the hydrogen phase, $\left(\mathrm{Fe}^{3+}\right)$ $\left[\mathrm{Fe}_{3 / 2}{ }^{3+} \mathrm{H}_{1 / 2}{ }^{+}\right] \mathrm{O}_{4}{ }^{2-}$, since the relative intensity of the $A$ - and $B$-site patterns is $1: 1.71 \pm 0.05$ and agrees with the 1:1.67 expected from (1).

However, the fraction of iron in (1) by weight is $69.9 \%$, compared to $56.9 \%$ found in our samples. Thermogravimetric and infrared measurements established that the samples contained water, adsorbed onto the particles' surface. Nevertheless, after allowing for the water, the spin-only moment expected from the observed cation distribution, assuming an antiparallel spin configuration for the $A$ and $B$ sublattices, is $76 \pm 5 \mathrm{emu} / \mathrm{g}$, still much greater than the observed value.

The reason for this discrepancy, readily apparent from Fig. 2(b), is that the spin arrangement in the ultrafine particles is certainly not collinear. If the iron spins were all parallel to the applied field, the $\Delta M=0$ transitions, lines 2 and 5 , would disappear. This is observed in bulk ferrites with the Néel spin configuration, ${ }^{8,9}$ including $\gamma-\mathrm{Fe}_{2} \mathrm{O}_{3} \cdot{ }^{4,7}$ In spite of the appreciable concentration of vacancies in the octahedral sites, there is no evidence of any canting in spectra of the bulk material in an applied field, and the average canting angle, if any, must be less than $15^{\circ}$. In the ultrafine particles the observed intensity ratio of the outer, middle, and inner pairs of lines is 3.00:0.69:1.08. A similar ratio is observed in an applied field of $25 \mathrm{kOe}$. Since $\mathrm{Fe}^{3+}$ is an $S$-state ion, the persistence of the middle pair cannot be due to crystalline anisotropy is an order of magnitude too small to account for the observations. ${ }^{4}$

Several interpretations of the observed intensity ratio are possible. For example, all the ions may be inclined to the direction of the applied field with an average angle of $32^{\circ}$, or else most of them may be aligned, but the remaining $34 \%$ may make random angles between 0 and $90^{\circ}$ or 0 and $180^{\circ}$. The latter possibility is rejected, because it would give a moment of $50 \mathrm{emu} / \mathrm{g}$. Of the other two, the possibility of a fraction making random angles between 0 and $90^{\circ}$ is likely to be closest to the truth. The percentage corresponds closely to the percentage in the surface layer, and a moment of $63 \pm 5 \mathrm{emu} / \mathrm{g}$ is predicted, in agreement with observation.

The surface cations will have various numbers of neighboring cations on both sublattices. Many ferrimagnetic compounds have strong antiferromagnetic $A-B$ exchange, and weaker, competing $A-A$ and $B-B$ interactions. The surface ions are analogous to the magnetic cations in ferrites and garnets with partial diamagnetic substitutions, which are canted from the direction of the net moment. ${ }^{10-13}$

The ultrafine particles, then, may be visualized as having a core with the normal spin arrangement, and a surface layer in which the spins of the ions are not oriented at random, but are inclined at some angle to their normal direction, which depends on their magnetic nearest neigh- 
bors. However, the possibility of some canting of the ions in the interior cannot be excluded, as there is likely to be an extra, random concentration of vacancies which can be appreciable in the smallest particles in the distribution. ${ }^{14}$

The author is grateful to Mrs. Dorothy Khalafalla for making most of the magnetization measurements and to Dr. I. Maartense for helpful discussions.

This work was supported by the National Research Council of Canada.

* Now at Laboratoire d'Electrostatique et du Physique du Métal, Cedex 166, 38-Grenoble Gare, France.

${ }^{1}$ A. E. Berkowitz, W J. Schuele, and P. J. Flanders, J. Appl. Phys. 39, 1261 (1968).

${ }^{2}$ D. E. Speliotis, J. R. Morrison, and G. Bate, in Proceedings of the Intermational Conference on Magnetism, Nottingham, England, 1964 (Institute of Physics and the Physical Society, London, England, 1964), p. 623.
${ }^{3}$ E. W. Gorter, J. Phys. Radium 12, 189 (1951).

${ }^{4} \mathrm{~J}$. M. D. Coey and D. Khalafalla, to be published.

${ }^{5}$ A. Aharoni, Phys. Rev. 135, A447 (1964), and 177, 793 (1969). A treatment of the relaxation time of superparamagnetic particles with cubic anisotropy by A. Aharoni and $\mathrm{R}$. Bijaoui is to be published.

${ }^{6}$ G. A. Ferguson and M. Hass, Phys. Rev. 112, 1130 (1958).

${ }^{7}$ J. M. D. Coey, A. H. Morrish, and G. A. Sawatzky, J. Phys. (Paris) Colloq. 32, C1-271 (1971).

${ }^{8}$ G. A. Sawatzky, F. van der Woude, and A. H. Morrish, Phys. Rev. 187, 747 (1969).

${ }^{9}$ J. Chappert and R. B. Frankel, Phys. Rev. Lett. 19, 570 (1967).

${ }^{10}$ N. S. Satya Murthy, M. G. Natera, S. I. Youssef, R. J. Begum, and C. M. Srivastava, Phys. Rev. 181, 969 (1969).

${ }^{11}$ J. M. Daniels and A. Rosencwaig, Can. J. Phys. $\underline{48}$, 381 (1970).

${ }^{12}$ S. Geller, H. J. Williams, G. P. Espinosa, and R. C. Sherwood, Bell Syst. Tech. J. 43, 565 (1964); S. Geller, J. Appl. Phys. 37, 1408 (1966).

${ }^{13}$ A. Rosencwaig, Can. J. Phys. 48, 2857, 2868 (1970).

${ }^{14}$ L. Neel, J. Phys. Soc. Jap. 17, Suppl. B1, 676 (1962).

\title{
Critical Magnetic Thermal Expansivity of $\mathrm{RbMnF}_{3}$
}

\author{
Brage Golding \\ Bell Laboratories, Murray Hill, New Jersey 07974
}

(Received 8 April 1971)

\begin{abstract}
Precision measurements of the magnetic thermal expansivity of $\mathrm{RbMnF}_{3}$ near its critcal (Neel) temperature are reported. The critical exponents are $\alpha=+0.007 \pm 0.02$ and $\alpha^{\prime}=-0.100 \pm 0.03$, in disagreement with scaling predictions. The rounding about $T_{c}$ due to sample inhomogeneity is observed to be asymmetric.
\end{abstract}

The experimental determination of the asymptotic temperature dependence of a magnetic solid's specific heat or thermal expansivity as its critical point is approached has proven extremely difficult. In contrast to the situation at the $\lambda$ point of liquid helium, ${ }^{1}$ or the gas-liquid critical point, ${ }^{2,3}$ the rounding near $T_{c}$ caused by the solid's inhomogeneity is large and poorly understood. ${ }^{4}$ The resultant ambiguity in locating $T_{c}$ and in determining the extent of the rounded region usually leads to large uncertainties in critical exponents. We report here measurements of the magnetic thermal expansivity of the isotropic cubic antiferromagnet $\mathrm{RbMnF}_{3}$ in the vicinity of its critical (Néel) temperature, $T_{c} \simeq 83 \mathrm{~K}$. As a result of the sensitivity and accuracy obtainable with capacitive dilatometry, as well as the perfection of our $\mathrm{RbMnF}_{3}$ crystal, ${ }^{5}$ we have been able to establish with a high degree of certainty the location of $T_{c}$ and the extent of the rounded re- gion about $T_{c}$. The region over which the magnetic thermal expansivity obeys a simple power law shows a pronounced asymmetry about $T_{c}$, with substantially more rounding occurring below $T_{c}$. A simple power law with critical exponents $\alpha$ $=+0.007$ and $\alpha^{\prime}=-0.100$ gives the best fit to the expansivity data.

A brief discussion of the experimental techniques has been presented previously. ${ }^{6}$ The magnetic part of the volume thermal expansivity ${ }^{7}$ (at constant pressure and zero magnetic field), $\beta_{m}$, is shown in Fig. 1 as a function of $\log _{10} \epsilon$, where $\epsilon \equiv\left|T-T_{c}\right| / T_{c}$. We shall be concerned with determining the values for the exponents $\alpha$ and $\alpha^{\prime}$ defined by the asymptotic forms $\mathrm{s}^{8,9}$

$$
\begin{aligned}
& \beta_{m}=(A / \alpha)\left(\epsilon^{-\alpha}-1\right)+B, \quad T>T_{c} ; \\
& \beta_{m}=\left(A^{\prime} / \alpha^{\prime}\right)\left(\epsilon^{-\alpha^{\prime}}-1\right)+B^{\prime}, \quad T<T_{c} .
\end{aligned}
$$

In practice, experimental data can be expected to 